\title{
Study of Solvent Effects in Diels-Alder Reaction through Charge Transfer Formation by Using Semi-empirical Calculations
}

\author{
Mehdi Salih Shilhab \\ Al-Nahrain University, College of Science, Department of Chemistry, Al-Jadrya, Baghdad-Iraq \\ E-mail: mehdi_shihab@yahoo.com \\ Received Februarv 15, 2008
}

\begin{abstract}
Study of computational model of the concerted Diels-Alder reaction between 9,10-diniethyl antluacene (as donor) and tetracyanoethylene (as acceptor) in absence and in presence of aromatic solvents (benzene, mesitylene and hexamethylbenzene, as donors) using an AM1 seni-empirical method. AM1 method used to study the neutral charge transfer complex models that could be expected between donor and acceptor during the course of the concerted Diels-Alder reaction. Calculated enthalpies of reaction of the charge transfer complexes models showed physical and chemical meaning for explain the effect of aromatic solvents on the kinetic process of concerted Diels-Alder reaction that contains tetracyanoethylene.
\end{abstract}

Key Words : AMl method, Neutral charge transfer conılex, Concerted Diels-Alder reaction

\section{Introduction}

The Diels-Alder reaction which involving [4+2] cycloaddition of diene to dienophile to give a six-member unsaturated ring, is occupied a wide space in the organic synthesis. Fukui and coworkers ${ }^{1}$ introduced the frontier electron theory by defining highest occupied molecular orbital (HOMO) and lowest unoccupied molecular orbital (LLMO). The energy difference between frontier orbitals HOMO and LUMO is known as HOMO-LLMO gap (HLG). The first type of HLG is called an intramolecular HLG within a single molecule, and this type of HLG does not sufficient to explain the chemical reactivity. ${ }^{2}$ The second type of HLG is called intermolecular HLG for energy difference between HOMO of one molecule and LUMO of another molecule of DielsAlder reaction, and this kind of HLG is applicable for interpreting the chemical reactivity of Diels-Alder reaction. " Figure 1 shows the two frontier orbital molecule energy levels of intramolecular and intermolecular HLG of DielsAlder reaction which proceeds under orbital control.

Diels-Alder reactions which are known to be far less solvent dependent, but it was found that the rate constant of some Diels-Alder reactions ${ }^{+}$which involving tetracyanoethylene as an acceptor, have solvent dependent. This solvent effect can be considered as solute-solvent interaction.

From another side, large of experimental evidences of charge transfer (CT) complexes had been reported in sold or in solution in different field of chemistry." According to Mulliken's theory, ${ }^{6}$ tormation of the (CT) complex involves transition of an electron from HOMO of donor to LUMO of acceptor. Opposing p systems typically adopt a parallelplaner (stacked or offset-stacked) geometry. The interaction between the donor and acceptor is characteristic by electronic absorption band with low energy. One of these mole-

Abbreviations: $D$, donor; $A$, acceptor; $\mathrm{IP}_{\mathrm{D}}$, ionization potential of electron donor; $E_{A}$, electron affinity of electron acceptor; $\phi$, dihedral angle; $d$, intennolecular distance; $\left(\Delta \mathrm{H}^{\prime}{ }_{\mathrm{f}}\right.$, kcalmol $\left.{ }^{-1}\right)$, heat of formation. cular complexes is $\pi, \pi$-complex between neutral molecules. ${ }^{57}$ In the present work, it was used synchronous concerted Diels-Alder reaction between a symmetric diene with strong $\pi$-donor 9,10-dimethylanthracene (DMA) $\left(\mathrm{IP}_{\mathrm{D}}=7.04 \mathrm{ev}^{8 \mathrm{a}}\right)$ and a symmetric dienophile with strong p-acceptor tetracyanoethylene (TCNE) $\left(\mathrm{E}_{\mathrm{A}}=2.88 \mathrm{ev}^{8 \mathrm{a}}\right)$. The reaction above (gas phase) proposed to proceed in aromatic solvents with different characterization of neutral planar $\pi$-donor (benzene (Ben), $\mathrm{LP}_{\mathrm{D}}=9.25 \mathrm{ev}^{8 \mathrm{l}}$; mesitylene (Mes), $\mathbb{P}_{\mathrm{D}}=8.14 \mathrm{ev}^{8 \mathrm{~b}}$; hexamethylbenzene $\left.(\mathrm{HMB}), \mathrm{IP}_{\mathrm{D}}=7.85 \mathrm{ev}^{8 \mathrm{c}}\right)$. Strong $\pi$ acceptor TCXE can be established a neutral $\pi, \pi$-nolecular

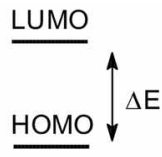

(a)

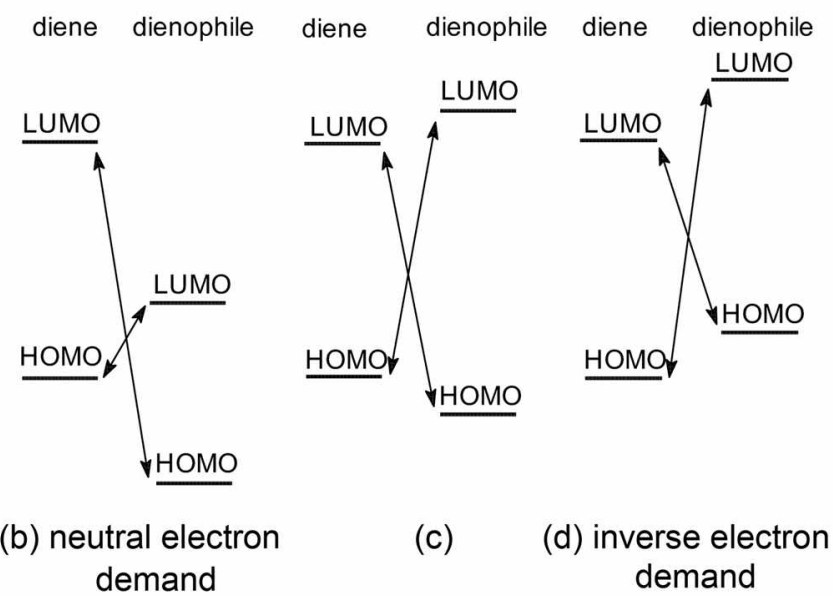

Figure 1. Controlling interactions of frontier orbital molecular energy of (a) intramolecular HOMO-LLMO gab; (b, c, d) intemolecular HOMO-LUMO gab for three type of Diels-Alder reaction. 

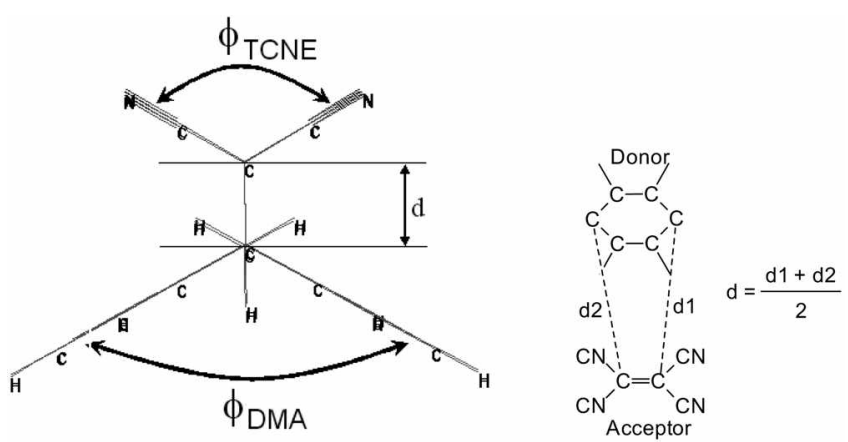

Figure 2. Dihedral angle (f) and intermolecular distance (d) are considered for geometry optimization for the system under study.

complex (MC) by situation to be juxtaposed on each one of $\pi$-donor molecules (DMA, Ben. Mes, HMB) and separated by an interplanar distance with different stabilization energies. It was possible to carry out comparative theoretical calculations on the Diels-Alder reaction between DMA and TCXE in the presence and without aromatic solvents (Ben. Mes, and HMB). The purpose of this work is to show solvent effects on the [2+4] cycloaddition reaction by using low cost of quantum chemical calculation level in gas phase.

\section{Computational Method}

All molecules structures of $\pi$-donors and $\pi$-acceptor were fully optimized by using $\mathrm{AM} 1^{9}$ semi-empirical method as implemented in the program package HyperChem 7.1 (Hypercube Inc. Gainsville FL.) with using default gradient methods. The geometry structure of lowest energy $\left(\Delta H_{\mathrm{f}}^{\mathrm{f}}\right)$ was considered as the equilibrium conformation. The optimization of the neutral $\pi, \pi$-molecular complexes were carried out from the optimized geometry of components, and consi- dering the intermolecular distance (d) as a free parameter. The product and the transition state (TS) of concerted DielsAlder reaction were characterized as the minima and saddle point as (TS) by frequency calculation. The intra- and intermolecular HLG could be found out by distortion of single molecule of DMA and TCNE through changing dihedral angle from $180^{\circ}$ to $110^{\circ}$ that accompanied changing in the HOMO and LUMO of DMA and TCNE (see Fig. 2).

\section{Results and Discussion}

Concerted Diels-Alder Reaction without Solvents. Generally, mechanism route of Diels-Alder reaction proceeds by approach reactants with each other by a way that makes the planes of each reactant are canted for easy overlap of $\pi$-electrons to form new bonds. ${ }^{14}$ By using AM1 semiempirical calculation, the optimized geometry was determined on DMA, TCNE, neutral $\pi, \pi$-MC(DMA-TCNE), TS(DMA-TCNE) and the product as shown in Figure 3.

For the optimization of the neutral $\pi, \pi$-MC(DMA-TC NE) in ground state, it was considered the position of DMA molecule to TCNE molecule under strong interaction to each other. The main factors for the formation of $\mathrm{MC}$ are CT interaction and intermolecular hindering. The face to face structure allows a CT interaction in neutral $\pi, \pi$-MC (DMATCNE), and stable geometry leads to have to be donor is approximately coplanar to acceptor. The synchronous saddle point is characterized as true TS by possessing one imaginary frequency, by using frequency calculation within AM1 level. The $\left(\Delta \mathrm{H}_{\mathrm{f}} \mathrm{f}\right)$ values of more stable geometry, is shown in the Figure 3, obtained for DMA $=55.2, \mathrm{TCNE}=152.3$, neutral $\pi, \pi$-MC(DMA-TCNE) $=206.4$, TS(DMA-TC $N E)=$ 244.8 and product $=203.5 \mathrm{kcal} \mathrm{mol}^{-1}$. The stabilization energy of neutral $\pi, \pi-\mathrm{MC}(\mathrm{DMA}-\mathrm{TCNE})$ is $-1.1 \mathrm{kcal} \mathrm{mol}^{-1}$

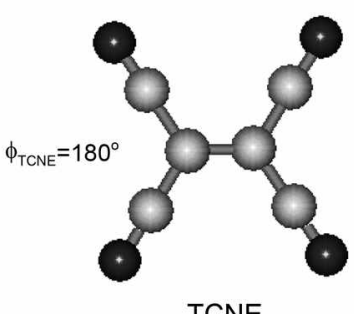

TCNE

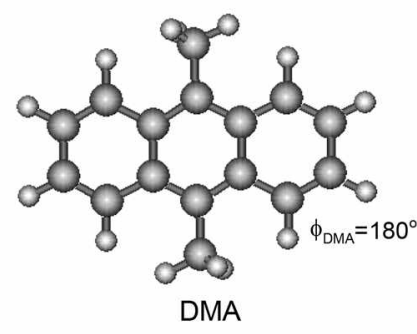

DMA

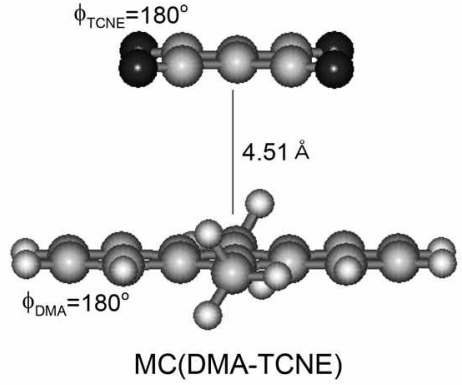

MC(DMA-TCNE)

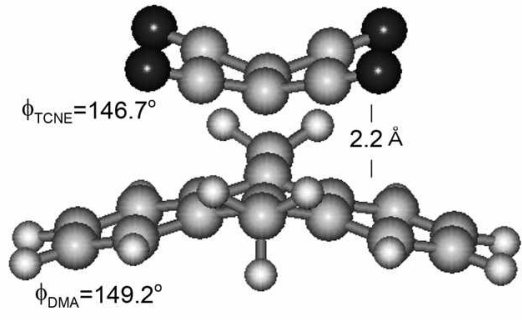

TS(DMA-TCNE)

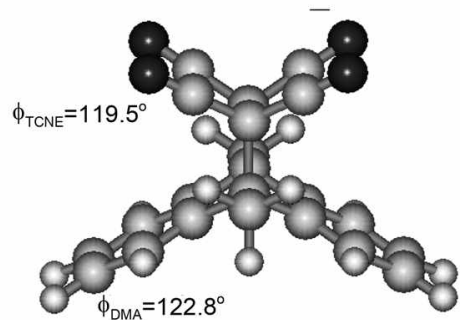

Product(DMA-TCNE)

Figure 3. Optimized geometry of reactants, neutral $\pi \cdot \pi$-molecular complex (MC). transition state (TS) and product (d $=1.5805 \AA$ ) of the concerted Diels-Alder reaction using AM1 level as implemented in the program package HyperChem version 7.1, showing dihedral angle ( $)$ and intemolecular distance (d). 


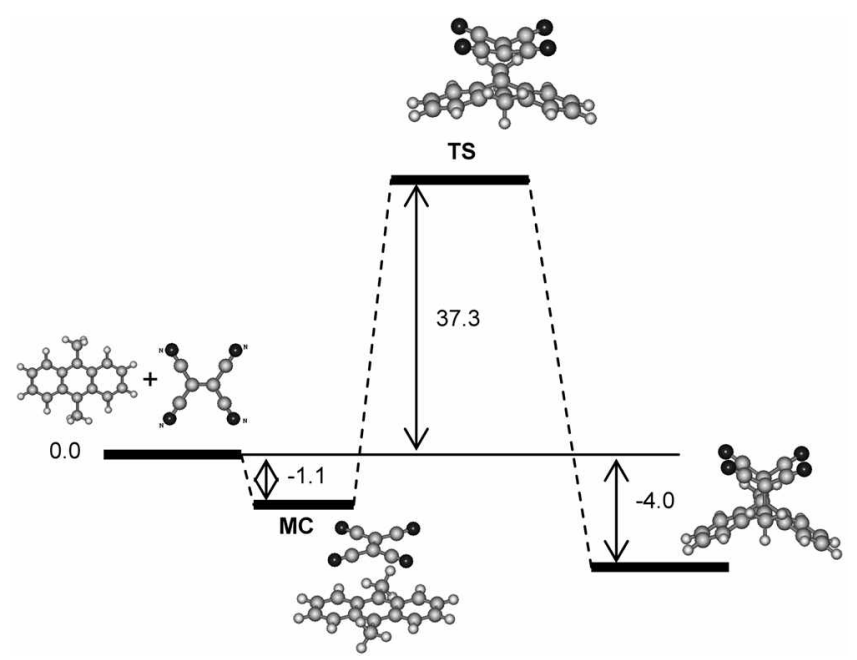

Figure 4. Relative energy diagram for the concerted Diels-Alder reaction between TCNE and DMA, energies are in $\mathrm{kcal} \mathrm{mol}^{-1}$.

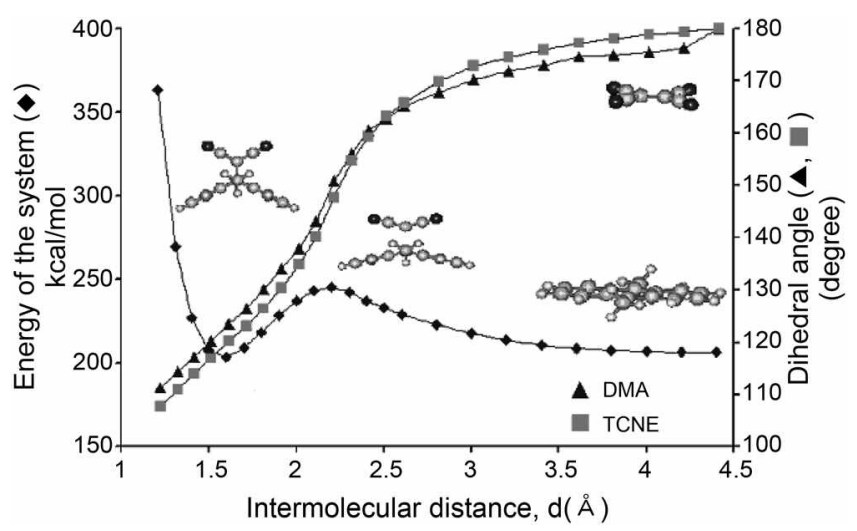

Figure 5. Changing of the energy of the system and the dihedral angles of DMA and TCNE by decreasing the intermolecular distance (d) using AM1 method.

within the experimental data. ${ }^{6}$ Figure 4 is displayed profile of relative energy of every step (MC, TS and product) in the reaction obtaining by subtracted from the sum of $\left(\Delta \mathrm{H}^{\mathrm{v}} \mathrm{f}\right)$ of isolated components. It's clear from Figure 4 . that the DielsAlder reaction proceeds in the beginning by $\mathrm{MC}^{10}$ which is more stable than reactant and concerted cycloaddition proceeds by interaction between two $\pi$-systems caused increasing energy until TS. Finally, formation new bonds (product) shift the system to the lowest energy.

It was also possible to explore the distortion of the DMA and TCXE during the concerted Diels-Alder reaction when DMA approach to TCNE until the formation of product by calculation of $\left(\Delta \mathrm{H}_{\mathrm{f}}^{\circ}\right)$ of the system and $\phi$, dihedral angle as function of intermolecular distance (d), as shown in Figure 5.

Figure 4 illustrates the changing of the energy system a long the concerted Diels-Alder reaction, with a peak of TS and a valley of the product and the distortion process of the TCXE and DMA during the reaction were near from each other and there is a crossing point and after that there is a slightly diversion. That because the system changes during the reaction from unsaturated reactants to saturated frag-

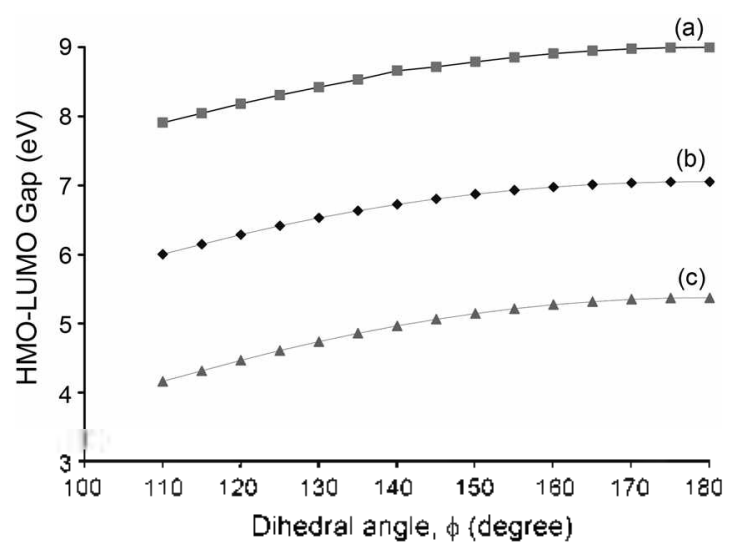

Figure 6. The intramolecular HLG of (a) TCNE, (b) DMA and (c) the intermolecular HLG of (HOMODNA-LUMOTCNE).

ments ( $\mathrm{sp}^{2}$ converted to $\mathrm{sp}^{3}$ ) at the formation of $\sigma$-bonds (product).

The values of intra- and intermolecular HLG for minima were calculated (AM1 method) by decreasing the dihedral angles from $180^{\circ}$ to $110^{\circ}$ for DMA and TCNE. The results revealed that both intra- and intermolecular HLG decrease with decreasing dihedral angle as shown in Figure 6.

From Figure 6, the decreasing dihedral angle of DMA and TCNE until about $150^{\circ}$ for both where the state activated complex makes redistribution the density of electrons around DMA and TCNE as shown in Figure 7, and here enhancing the polarizabilty for both by increasing the energy level of HOMODM and decreasing the energy level of LUMO $_{\text {TCNE }}$ that causes increasing the reactivity of reactants.

Finally, the energy of frontier orbitals of DMA, TCNE, $\pi, \pi$-MC(DMA-TCNE), TS(DMA-TCNE) and the product are shown in the Table 1. And this table shows that the reaction between DMA and TCYE is neutral electron demand because the difference HOMO ${ }_{\text {DMA }}-\mathrm{LUMO}_{\mathrm{TCNE}}=$ $5.3716(\mathrm{eV})$ and the difference $\mathrm{HOMO}_{\mathrm{TCNE}}-\mathrm{LUMO}_{\mathrm{DMA}}=$ $10.6800(\mathrm{eV})$.

Neutral $\pi, \pi$ Molecular Complexes. All molecules strictures of p-donors (Ben, Mes, HMB) were fully optimized by

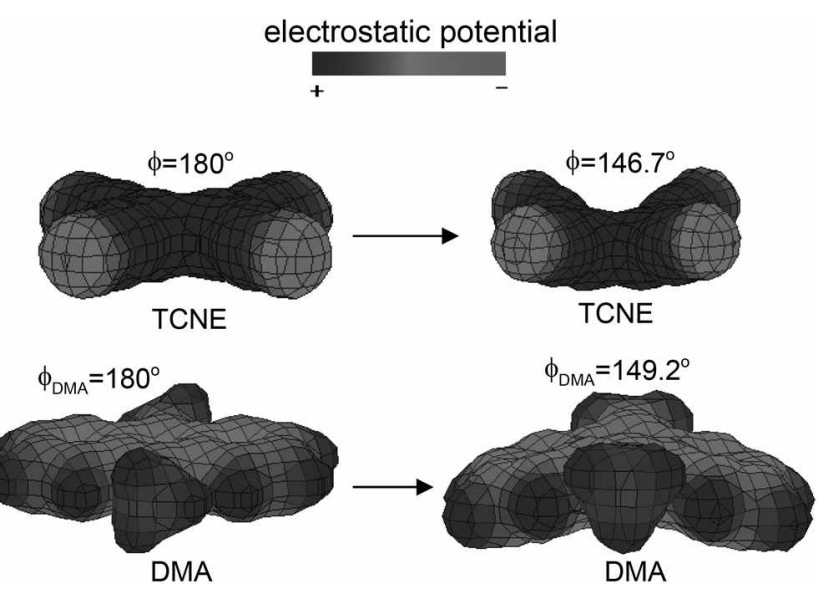

Figure 7. Artificially decreasing the DMA and TCNE from $180^{\circ}$ to about $150^{\circ}$ (where TS zone) illustrates the changing electron density around both of them, using AM1 method. 
Table 1. Frontier orbital energies (in eV) and intramolecular HLG (in $\mathrm{eV}$ ) of DMA. TCNE, $\pi$-MC(DMA-TCNE). TS(DMA-TCNE) and the product caleulated from AM1 level

\begin{tabular}{llrc}
\hline \multicolumn{1}{c}{ Molecule } & Orbital & Energy & intramolecular HLG \\
\hline \multirow{2}{*}{ DMA } & HOMO & -7.8963 & 7.0638 \\
& LUMO & -0.8425 & \\
\multirow{2}{*}{ TCNE } & HOMO & -11.5225 & \multirow{2}{*}{8.9978} \\
& LUMO & -2.5247 & \\
\multirow{2}{*}{$\pi . \pi$-MC(DMA-TCNE) } & HOMO & -8.0472 & \multirow{2}{*}{5.7264} \\
\multirow{2}{*}{ TS(DMA-TCNE) } & HOMO & -2.3208 & \\
\hline \multirow{2}{*}{ Product } & LUMO & -1.5680 & 7.2816 \\
& HOMO & -9.8572 & \multirow{2}{*}{9.4225} \\
\hline
\end{tabular}

using AM1 (see Fig. 8A). We suggested that the optimization geometry of neutral $\pi, \pi$-molecular complexes in ground state are including two kinds of MC. First (D-A) dyad $\pi, \pi-\mathrm{MC}$ and second (D-A-D) triad $\pi, \pi-\mathrm{MC}^{\prime l}$ (see Fig. 8 for $\mathrm{B}$ and $\mathrm{C}$, respectively). $\pi, \pi$-MC had been mentioned in different reports under the solvents effect on equilibrium constant of complexes formation ${ }^{1286}$ and on rate constant and equilibrium constant of some Diels-Alder reactions. ${ }^{\text {tc }}$

This work is suggesting these kinds of neutral $\pi, \pi$ - molecular complexes and the main factors for the formation of $\mathrm{MC}$ are $\mathrm{CT}$ interaction and intermolecular hindering. The face to face structure allows a CT interaction in neutral $\pi, \pi$ $\mathrm{MC}$, and stable geometry leads to have to be donor is approximately coplanar to acceptor by using AM1 method as a tool for geometry optimization. The intermolecular distance (d) at equilibrium for all the (D-A) dyad $\pi, \pi-\mathrm{MC}$. and (D-A-D) triad $\pi, \pi-\mathrm{MC}$ are showing in the Fig $(2,7)$ are with the range observed in the organic CT complexes. ${ }^{6}$ The values of $\left(\Delta \mathrm{H}^{\circ} \mathrm{f}\right)$ and the energy of frontier orbitals of $\mathrm{HMB}$, MES and Ben and also (D-A) dyad $\pi, \pi$-MC and (D-A-D) triad $\pi, \pi$-MC as demonstrating in the Table 2 and Table 3 , respectively.

The Table 3 shows that the stabilization energy of neutral $\pi, \pi$-MC within the range of the reaction enthalpies for the formation of the neutral aromatic $\pi, \pi$-MC which are $0 \ldots-5$ $\mathrm{kcalmol}^{-1} .^{13}$ And also Table 3 shows that the properties of $\mathrm{MC}$ formation (4-6 and 8-13) decreases when the reaction proceeding from the $\mathrm{MC}$ form passing by $\mathrm{TS}$ and product.
(A)

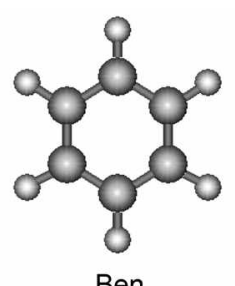

(B)

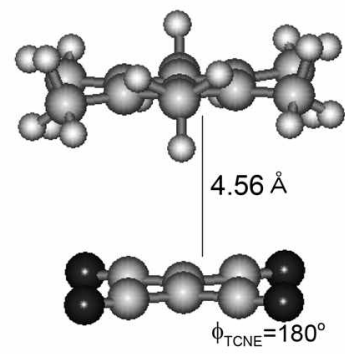

MC(HMB-TCNE)

(C)

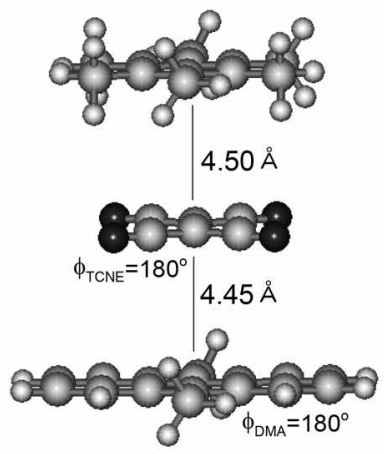

MC(DMA-TCNE-HMB)

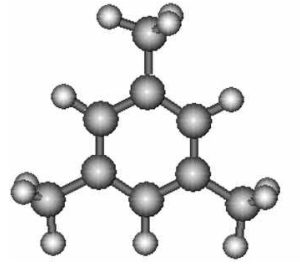

Mes

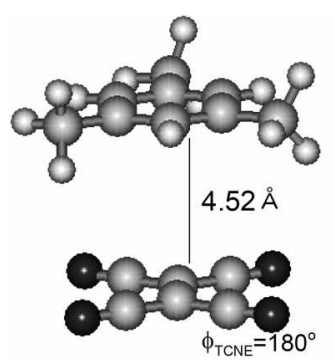

MC(Mes-TCNE)

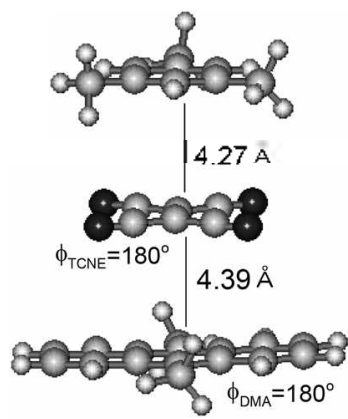

MC(DMA-TCND-Mes)
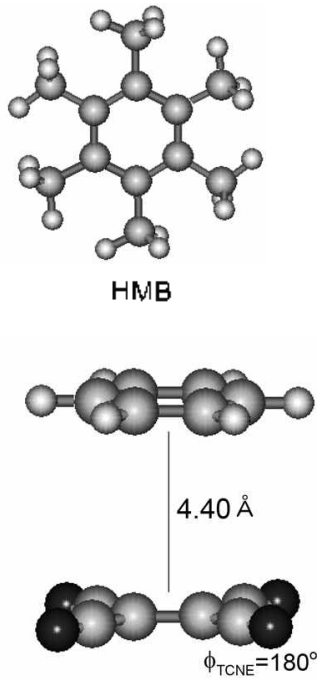

MC(Ben-TCNE)

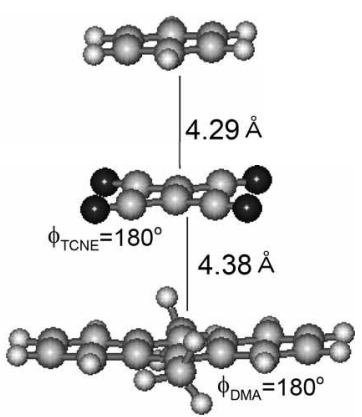

MC(DMA-TCNE-Ben)

Figure 8. (A) Optimized geometry of reactants (Ben, Mes, HMB); (B) Optimized geometry of (D-A) dyad $\pi, \pi$-molecular complexes [MC(HMB-TCNE). MC(Mes-TCNE), MC(Ben-TCNE)], opposing $\pi$ systems typically adopt a parallel-planer geometry and showing dihedral angle (f) and intermolecular distance (d); (C) Optimized geometry of (D-A-D) triad $\pi, \pi$-molecular complexes [MC(DMA-TCNEHMB), MC(DMA-TCNE-Mes): MC(DMA-TCNE-Ben)]. opposing $\pi$ systems typically adopt a parallel-planer geometry and showing dihedral angle (f) and intemolecular distance (d). Using AMl level as implemented in the program package HyperChem version 7.1. 
Table 2. Frontier orbital energies (in eV) and $\Delta \mathrm{H}^{\circ}{ }_{\mathrm{r}} \mathrm{kcal} \mathrm{mol}{ }^{-1}$ of HMB. Mes and Ben were caleulated from AM1 level

\begin{tabular}{|c|c|c|c|c|}
\hline Molecule & $\Delta \mathrm{H}^{\prime}$ & Orbital & Energy & HOMO-LUMO \\
\hline \multirow{2}{*}{$\mathrm{HMB}$} & \multirow{2}{*}{-13.1} & HOMO & -8.7510 & \multirow{2}{*}{9.3266} \\
\hline & & LUMO & 0.5756 & \\
\hline \multirow{2}{*}{ Mes } & \multirow{2}{*}{0.9} & HOMO & -9.1529 & \multirow{2}{*}{9.7186} \\
\hline & & LUMO & 0.5657 & \\
\hline \multirow{2}{*}{ Ben } & \multirow{2}{*}{21.9} & HOMO & -9.6529 & \multirow{2}{*}{10.2078} \\
\hline & & LUMO & 0.5549 & \\
\hline
\end{tabular}

Table 3. $\left(\Delta \mathrm{H}_{\mathrm{f}}^{\mathrm{O}} \mathrm{kcalmol} \mathrm{k}^{-1}\right)$ and frontier orbital energies (in eV) of (D-A) dyad neutral $\pi, \pi$-MC and (D-A-D) triad neutral $\pi, \pi$-MC and others were calculated from AMI level

\begin{tabular}{|c|c|c|c|c|c|c|}
\hline & Interaction form & $\Delta \mathrm{H}^{\circ}$ & Orbital & Energy & $\Delta \mathrm{E}$ & $\begin{array}{l}\text { Stabili } \\
\text { zation } \\
\text { energy }\end{array}$ \\
\hline \multirow{2}{*}{1} & $\mathrm{MC}(\mathrm{HMB}-$ & \multirow{2}{*}{136.4} & HOMO & -8.9336 & \multirow{2}{*}{6.5535} & \multirow{2}{*}{-2.9} \\
\hline & TCNE) & & LUMO & -2.3801 & & \\
\hline \multirow{2}{*}{2} & MC'Mes- & \multirow{2}{*}{150.4} & HOMO & -9.3607 & \multirow{2}{*}{6.9608} & \multirow{2}{*}{-1.0} \\
\hline & TCNE) & & LUMO & -2.3999 & & \\
\hline \multirow{2}{*}{3} & $\mathrm{MC}(\mathrm{Ben}-$ & \multirow{2}{*}{173.5} & HOMO & -9.8889 & \multirow{2}{*}{7.4592} & \multirow{2}{*}{-0.8} \\
\hline & TCNE) & & LUMO & -2.4297 & & \\
\hline \multirow{2}{*}{4} & MC(DMA- & \multirow{2}{*}{190.7} & HOMO & -8.0154 & \multirow{2}{*}{5.8397} & \multirow{2}{*}{-3.6} \\
\hline & TCNE-HMB) & & LUMO & -2.1757 & & \\
\hline \multirow{2}{*}{5} & MC(DMA- & \multirow{2}{*}{205.0} & HOMO & -8.0238 & \multirow{2}{*}{5.8403} & \multirow{2}{*}{-1.5} \\
\hline & TCNE-Mes) & & LUMO & -2.1835 & & \\
\hline \multirow{2}{*}{6} & MC(DMA- & \multirow{2}{*}{227.9} & HOMO & -8.0296 & \multirow{2}{*}{5.8091} & \multirow{2}{*}{-1.4} \\
\hline & TCNE-Ben) & & LUMO & -2.2205 & & \\
\hline \multirow{2}{*}{7} & MC(DMA- & \multirow{2}{*}{206.4} & HOMO & -8.0472 & \multirow{2}{*}{5.7264} & \multirow{2}{*}{-1.1} \\
\hline & TCNE) & & LUMO & -2.3208 & & \\
\hline \multirow{2}{*}{8} & TS(DMA- & \multirow{2}{*}{230.2} & HOMO & -8.5299 & \multirow{2}{*}{7.0475} & \multirow{2}{*}{-1.5} \\
\hline & TCNE) - $\mathrm{HVB}^{\prime \prime}$ & & LUMO & -1.4824 & & \\
\hline 9 & TS(DMA- & 244.1 & HOMO & -8.8038 & 73080 & 0.2 \\
\hline & TCNE) - Mes ${ }^{\prime \prime}$ & & LUMO & -1.4958 & & \\
\hline 10 & TS(DMA- & 266.9 & HOMO & -8.8063 & 7.3042 & 0.2 \\
\hline & TCNE) - Ben" & & LUMO & -1.5021 & & \\
\hline 11 & Product + HYR ${ }^{k}$ & 1887 & HOMO & -8.4911 & 80086 & $-17+2+3$ \\
\hline & 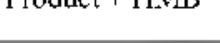 & 100.3 & LUMO & -0.3925 & 0.0700 & -1.3 \\
\hline 12 & Product - Mes ${ }^{h}$ & 202.5 & HOMO & -8.9017 & 8.5028 & 0.0 \\
\hline & & & LUMO & -0.3989 & & \\
\hline 13 & Product - Ben & 225.4 & HOMO & -8.4019 & 79991 & 0.0 \\
\hline & & & LUMO & -0.4028 & & \\
\hline
\end{tabular}

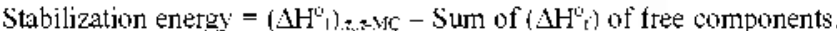
$\Delta \mathrm{E}=(\mathrm{HOMO}-\mathrm{LUMO})$ of MC. "Stabilization energy $=\left(\Delta \mathrm{H}^{\prime}\right)$ of TS (DMA - TCNE) + (Ben, Mes or HMB) - Sum of $\left[\left(A H^{\prime}\right)\right.$ of TS (DMA + $T C N E)+\left(\Delta H^{\circ}\right)$ of $($ Ben, Mes or $\left.\mathrm{HMB})\right]$. "Stabilization energy $=\left(\Delta \mathrm{H}^{\circ}\right)$ of product $+(B$ en. Mes or $\mathrm{HMB})-\mathrm{Sum}$ of $\left[\left(\Delta \mathrm{H}^{\circ}\right)\right.$ of product $+\left(\Delta \mathrm{H}^{\circ}\right)$ of (Ben, _Mes or HMB)]

Only in the case of $\mathrm{HMB}$ that the value of stabilization energy in TS and product is still considerable value. Therefore, we expected that the reaction of DMA with TCXE in the presence of HMB will not occur at all!

Concerted Diels-Alder Reaction in the Presence of Solvents. The interaction of acceptor (A) with the electron pair donor (D) is a result of an overlap of orbitals of two molecules, consequently, a finite electron density is created between the two partners according to the Eq. (1).

$$
\mathrm{D}:+\mathrm{A} \rightleftharpoons \mathrm{D}^{-\mathrm{F}}-\mathrm{A}=
$$

In this work the Diels-Alder reaction occurs as follow:

$\mathrm{TCNE}+$ aromatic solvent $\rightarrow \pi, \pi$-MC (colored solution) - DMA Product (colorless solution) $\leftarrow$ color solution gradually disappears

The equation of the proceeding Diels-Alder reaction in the presence of aromatic solvent can be illustrated by electrostatic potential view in the Scheme 1 where the redistribution of electron density in the donor and acceptor a long the Diels-Alder reaction.

This Scheme 1 can be supported by the values of stabilization energy and values of $\mathrm{DE}$ of Table 3. It could be compared between the values of stabilization energy and values of $\Delta E$ (electronic transitions) of Table 3 for (D-A) dyad neutral $\pi, \pi$-MC $(1-3,7)$ and (D-A-D) triad neutral $\pi, \pi$ $\mathrm{MC}(4-6)$, all these neutral $\pi, \pi-\mathrm{MCs}$ are in intreaction between the HOMO of donor and the LUMO of acceptor resulting in the stabilaization and formation of the CT complexes, in agreement with Mulliken's theory because the differenace between HOMO and LUMO in the neutral $\pi, \pi$ molecular complexes are small comparing with isolated components, as showning in the Figure 9.

To compare occurrence of concerted Diels-Alder reaction between DMA and TC NE in the presence and in the absence of aromatic solvent, It was also possible to calculate the distortion of the DMA and TCNE during the concerted Diels-Alder reaction when DMA approach to TCNE until the formation of product by calculation of $\left(\Delta \mathrm{H}_{\mathrm{f}}^{\mathrm{b}}\right)$ of the system as function of intermolecular distance (d), as shown in Figure 10.

Figure 10 shows the energy of the present system in the case of HMB more stable than the other solvents and also more stable than the system without solvent. The important step in the Figure 10 , its neutral $\pi, \pi-\mathrm{MC}$ because the stability of this step will influence on the stability of the other steps of TS and product. The high stability of neutral $\pi, \pi$-MC makes probability is weak to form TS (DMATCNE) and then product. Whereas, the distortion of the DMA and TCNE during the concerted Diels-Alder reaction when DMA approach to TCXE until the formation of product by calculation, $\phi$, dihedral angle as function of intermolecular distance (d), as shown in Figure 4, these distortions ( $\phi$ as function of $d$ ) for DMA and TCXE are still also unchanged in the presence of aromatic solvent (Ben, Mes, HMB).

Table 3, Scheme 1 and Figures 9, 10 give information about the stabilities of (D-A-D) triad neutral $\pi, \pi$-MC type (4, 5 and 6 in Table 3 ) which have low stabilization energies, and also the Table 3 shows the order of energy stability among (D-A-D) triad neutral $\pi, \pi$-MC type, where MC(DMATCNE-HMB) more stable then MC(DMA-TCXE-Mes) and the last MC(DMA-TCNE-Ben). That explains that MC(DMATCNE) needs different energies to separate aromatic molecules (HMB, Mes and Ben) in order from the product of 


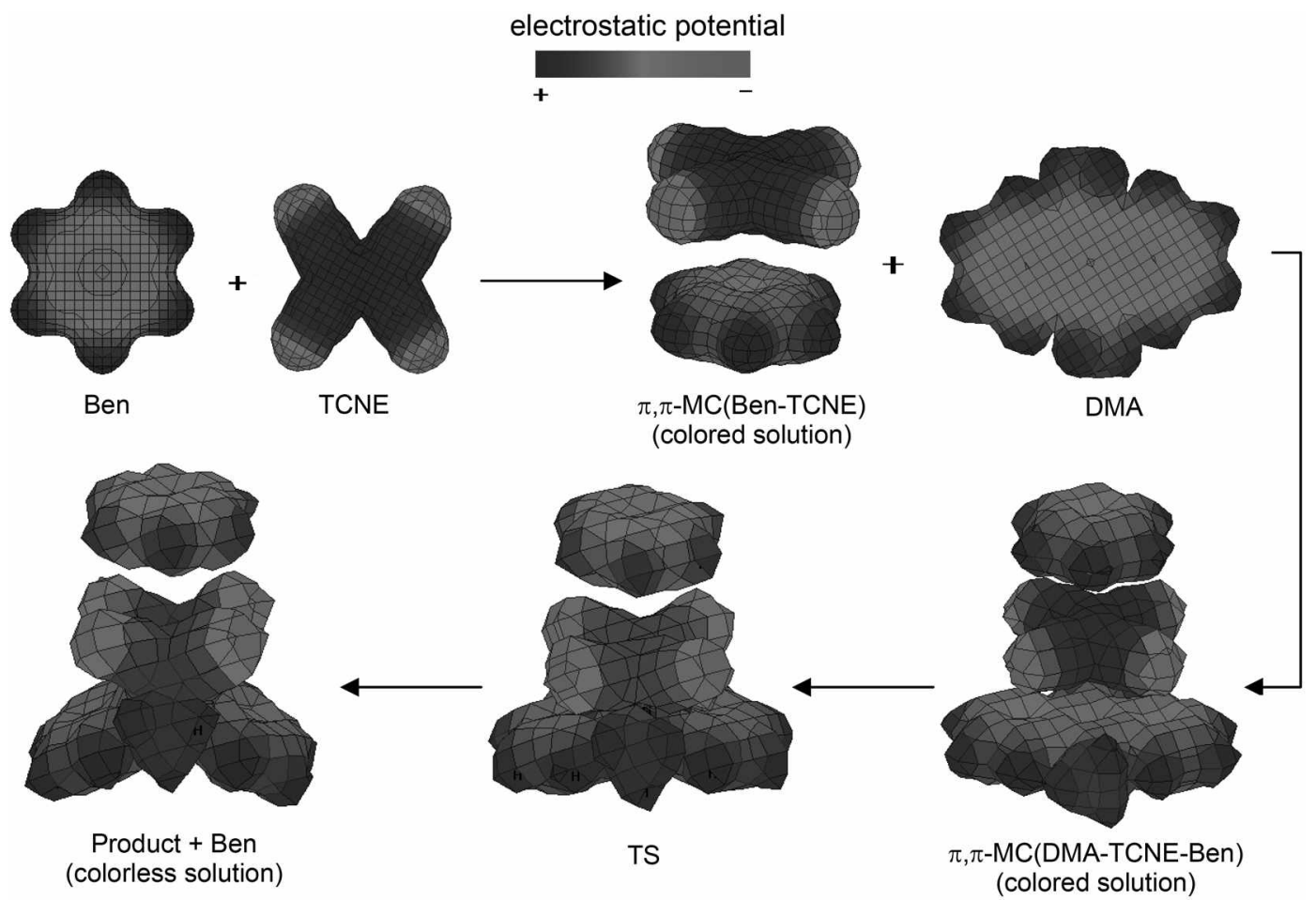

Scheme 1

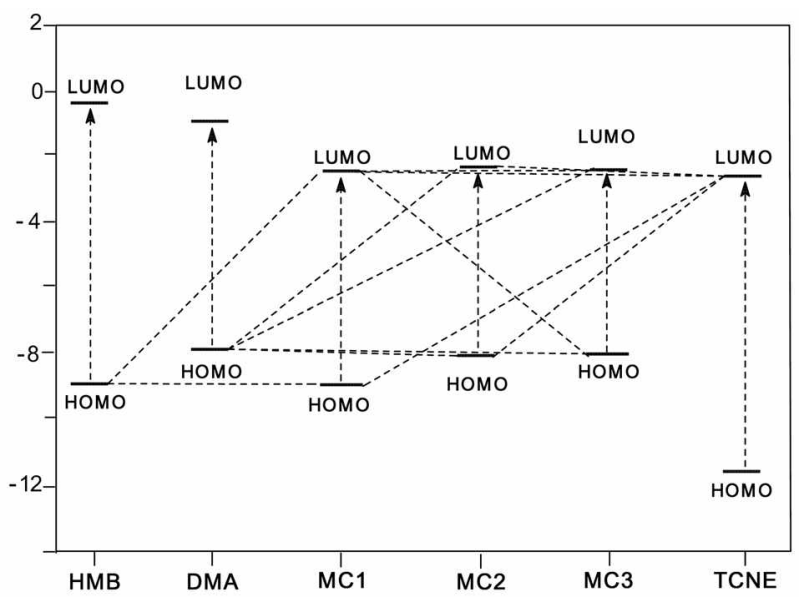

Figure 9. Conelation diagram for the formation of the MC l[HMBTCNE]. MC2[DMA-TCNE] from the components and formation of MC3[-DMA-TCNE-HMB] from DMA (as donor) and MC 1 (as acceptor).

concerted Diels-Alder reaction. That's it could be clear from the steps of Scheme 1 and changing the data of TS and product of (8-13) in the Table 3 and also the changing of energy of the system during the course of Diels-Alder reaction in the cases of TS and product as shown in the Figure 10. Increasing the stability of the (D-A-D) triad neutral $\pi, \pi$-MC type makes the formation of TS of DielsAlder reaction to be late and the rate of reaction will be more slow. That we could find in the effect of solvent on the rate of the reaction of anthracene with TCNE in different aromatic solvents. ${ }^{\text {tc }}$

In the Figure 11 shows the Optimized geometry of the

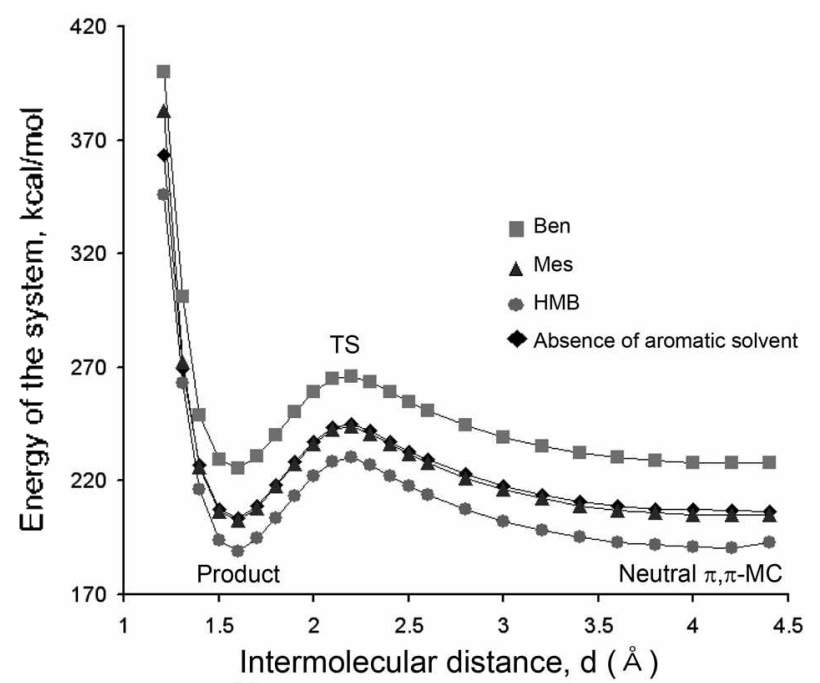

Figure 10. Changing of the energy of the system of DMA and TCNE by decreasing the intemolecular distance (d) in the presence and absence of aromatic solvent (Ben. Mes. HMB) using AM1 method.

interaction form of (Ben, Mes, HMB) with TS(DMATCNE) and with product of concerted Diels-Alder reaction between DMA and TCXE, and the intermolecular distance (d) increases between the aromatic solvents (Ben, Mes or $\mathrm{HMB}$ ) and TS form or product form with the decreasing the intermolecular distance (d) between the DMA and TCNE. That explains steps of separating aromatic solvents from the (D-A-D) triad neutral $\pi, \pi-\mathrm{MC}$ type to form the TS and product only between DMA and TCNE. Besides, the steric hindrance plays clear role on the intermolecular distance (d) for (D-A-D) triad neutral $\pi, \pi$-MC of A and B in the Figure 9. 

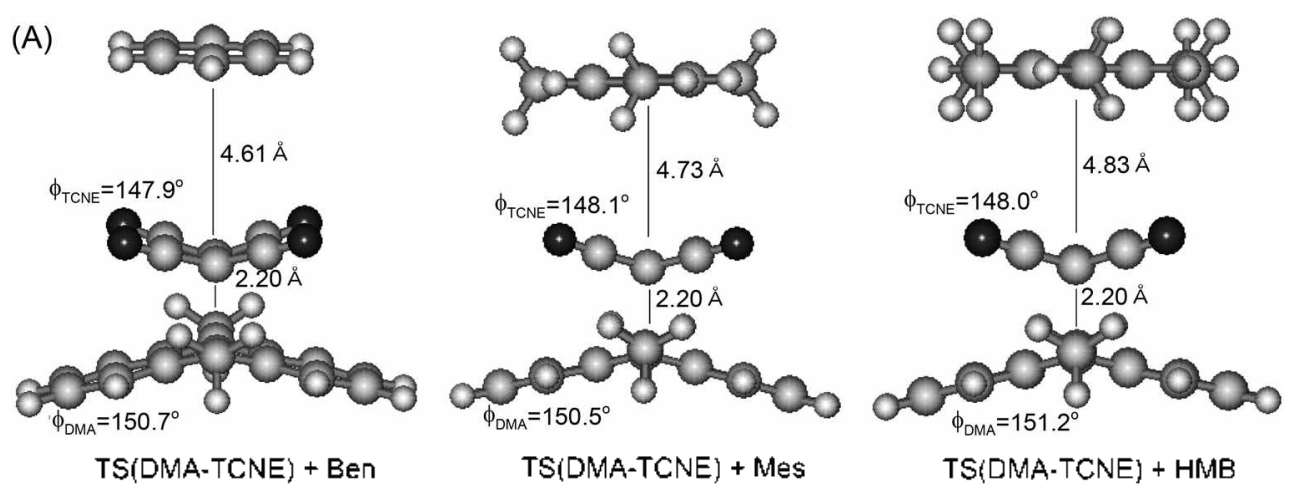

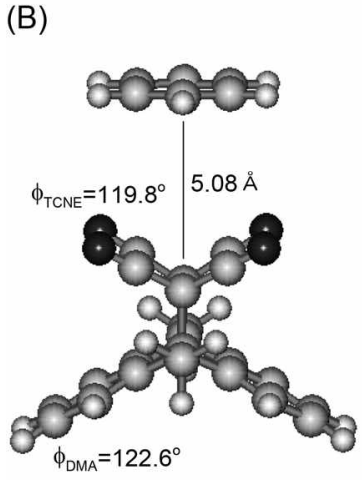

Product + Ben

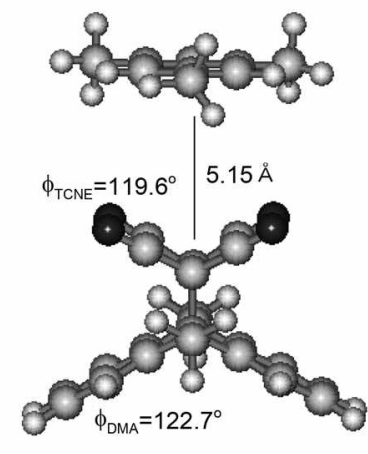

Product + Mes

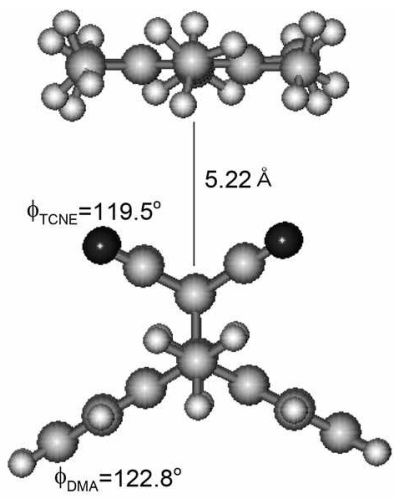

Product + $\mathrm{HMB}$

Figure 11. (A) Optimized geometry of the interaction form of (Ben, Mes. HMB) with TS(DMA-TCNE); (B) Optimized geometry of the interaction fonm of (Ben. Mes. HMB) with product $(d=1.5805 \AA$ ) of Diels-Alder reaction between DMA and TCNE. In these interactions each aromatic ring of (Ben. Mes, HMB) has opposite position to molecule of TS or product and showing dihedral angle (f) and intermolecular distance (d). Using AM1 level as implemented in the program package HyperChem version 7.1.

\section{Conclusion}

AMl of semi-empirical calculation could be a good tool to study the solvent effects in the Diels-Alder reaction between DMA and TCNE through the formation of different types of CT molecular complexes. Our present work suggested (DA) dyad neutral $\pi, \pi-\mathrm{MC}$ and (D-A-D) triad neutral $\pi, \pi-\mathrm{MC}$ and others and their chemical and physical properties were calculated from AMl level. These types of CT complexes can play important role by effecting of the rate on proceeding of Diels-Alder reaction which containing TC NE.

\section{References}

1. (a) Fujitomo, H.; Inagaki, S.; Fukui, K. $J$. Am. Chem. Soc. 1976 , 98, 2670. (b) Fukui, K. Science 1982, 218,747.

2. (a) Haddon, R. C.; Fukunaga, T. Tetrahedron Lett. 1980, 21, 1191. (b) Hess, B. A., Ir; Schaad, L. I. J. Am. Chem. Soc. 1971, 93, 2413. (c) Zhou, Z.; Рar, R. G. J. Am. Chem. Soc. 1989, 11 , 7371 .

3. (a) Okanoto, Y. J. Phis. Chem. B 2001, 105, 1813. (b) Spino, C.; Rezaei, H.; Dory, Y. L. J. Org. Chen. 1998, 63, 5350.

4. (a) Andrews, L. J.; Keefer, R. M. J. An. Chent. Soc. 1955, 75, 3776. (b) Oshima, T.; Arikata, S.; Nagai, T. $J$. Chem. Res. 1981, (S) 204, (M) 2518. (c) Kiselev, V. D.; Konovalov, A. I.; Asano, T.;
Kashaeva, E. A.; Iskhakova, G. G; Shihab, M. S.; Medvedeva, M. D. J. Phys Org. Chem 2001, 14,636.

5. (a) Hobza, P.; Zaliadnik, R. Intermolecular Compleres; Elsevier: Amsterdan, 1988. (b) Foster, R. Organic Charge-Transfer Complexes; Academic press: London, New York, 1969; Holecular Complexes, Foster, R., Ed.; Elek Science: London, 1974/73; Vol. 1 \& 2. (c) Gavezzotti, A. Chent. Phis. Lett. 1989, 161, 67. (d) Gavezzotti, A.; Desiraju, G R. Acta Crustallogr: Sect. B Strict. Sci. 1988, 44, 427.

6. Mulliken, R. S.; Person, W. B. Molecular Complexes; Wiley Interscience: New York, 1969

7. Kobinata, S.; Nagakura, S. J. An. Chen. Soc. 1966, 88, 3905.

8. (a) Konovalov, A. I.; Kiselev, V. D. Russ. Chem. Bull., In. Ed. 2003, 52, 293. (b) Merrifield, R. E.; Philips, W. D. J. An. Chen. Soc. 1958, 80, 2778. (c) Howell, J. O.; Goncalves, J. M.: Amatore, C.: Klasinc, L.; Wightman, R. M.; Kochi, J. K. J. Am. Chem. Soc. 1984, 106, 3968.

9. Dewar, M. J. S.; Zoebisch, E. G; Healy, E. F.; Stewart, I. I. P. . An. Chem. Soc. 1985, 107, 3902

10. Kiselev, V. D.; Miller, J. G J. An. Chem. Soc. 1975, 97, 4036

11. Greer, L. M.; Blackstock, S. C. J. Org. Chem. 1996, 61, 7895 .

12. Thompson, C. C.; de Maine, P. A. D. J. Phis. Chem 1966, 69, 2766.

13. Reichardt, C. Solvents and Solven Effects in Organic Chentistry; Wiely-VCH: 2003; Chap. 2.

14. Houk, K. N.; Li, Y.; Evenseck, I. D. Angen: Chen. Int. Ed. Engl. 1992, 31, 682. 